\title{
Endoscopic Removal of Proximally Migrated and Embedded Pancreatic Stent: A Case and Review
}

\author{
Yao Weijie MD ${ }^{1}$, Ma Shiyang $\mathrm{MD}^{2}$, Bo Wenping $\mathrm{BS}^{1}$, Leng Junzhi $\mathrm{MD}^{1}$ and Wang Zuozheng $\mathrm{MD}^{1 *}$ \\ ${ }^{1}$ Department of Hepatobiliary Surgery, General Hospital of NingXia Medical University, China \\ ${ }^{2}$ Department of Gastroenterology, the Second Affiliated Hospital of Xi'an Jiaotong University, China
}

*Corresponding author: Zuozheng Wang, Department of Hepatobiliary Surgery, General Hospital of Ningxia Medical University,

Address: No. 804, Shengli South Street, Xingqing District, Yinchuan, Ningxia, China; 710004.

\section{ARTICLE INFO}

Received: 幽 June 11, 2020

Published: 幽 June 22, 2020

Citation: Yao Weijie MD, Ma Shiyang MD2, Bo Wenping BS, Leng Junzhi MD, Wang Zuozheng MD. Endoscopic Removal of Proximally Migrated and Embedded Pancreatic Stent: A Case and Review. Biomed J Sci \& Tech Res 28(3)-2020. BJSTR. MS.ID.004649.

\section{ABSTRACT}

Unfortunately, proximal stent migration into the pancreatic duct (PD) is not infrequent and often difficult to resolve. The authors report a case of successful removal of a proximally migrated PD stent that was also partially embedded into the pancreatic parenchyma after two months of migration.

Keywords: Pancreatic Stent; Proximal Migration; Removal; Embedding; Forceps; Endoscopy

\section{Introduction}

Pancreatic Duct (PD) stenting is commonly practiced during Endoscopic Retrograde Cholangiopancreatography (ERCP) for various pancreatic interventions and to reduce Post-ERCP Pancreatitis (PEP) risk. Unfortunately, proximal stent migration into the PD is not infrequent and often difficult to resolve. The authors report a case of successful removal of a proximally migrated PD stent that was also partially embedded into the pancreatic parenchyma.

\section{Case Report}

A 76-year-old man was admitted to an outside hospital with epigastric pain and obstructive jaundice. Abdominal Computed Tomography (CT) showed pancreatic necrosis, peri-pancreatic inflammation, bile duct and PD dilatation. ERCP was performed for suspected choledocholithiasis which was confirmed. On brief pancreatogram, the main PD was not dilated $(\leq 3 \mathrm{~mm})$. A single pigtail PD stent ( $5 \mathrm{~F} \times 6 \mathrm{~cm}$, Cook Medical, Winston-Salem, NC, USA) placement was attempted for PEP prophylaxis. Unfortunately, due to patient's sudden hiccups, the PD stent migrated proximally into the pancreas during deployment. Repeated attempts to remove the stent using wire guide cannulation through the stent, balloon and basket sweeps failed to remove the migrated stent. To avoid a prolonged procedure, a second PD stent was successfully placed (Figure 1). After discharge, two months later, the patient was admitted to our hospital for epigastric pain. The CT scan and X-ray showed a pigtail stent was embedded in the pancreatic parenchyma in the head (Figure 2). During ERCP, the second PD stent was easily removed. However, the migrated stent could not be removed using a disposable snare (SD-230U-20, Olympus, Japan) and a mini stone retrieval basket (MB5-1, Cook Medical) due to PD obstruction from the migrated stent and stent embedment. The distal end of stent was about $1 \mathrm{~cm}$ away from main PD orifice, and the forceps was thought to be the best option to access the stent. The authors attempted to manipulate the guide wire and dislodge the stent. However, the stent was found embedded firmly. The authors advanced the guide wire to the middle part of the stent and straightened the main PD. Endoscopic forceps (QYQ-AXC, HuiChun LTD, ChangZhou, China) was inserted into the PD next to a wire guide and captured the distal end of the migrated stent. The stent was then entirely removed. A new PD stent was placed for PEP prophylaxis. In the next morning, The patient's serum amylase returned within normal limits the next day and there was no procedure complication. 


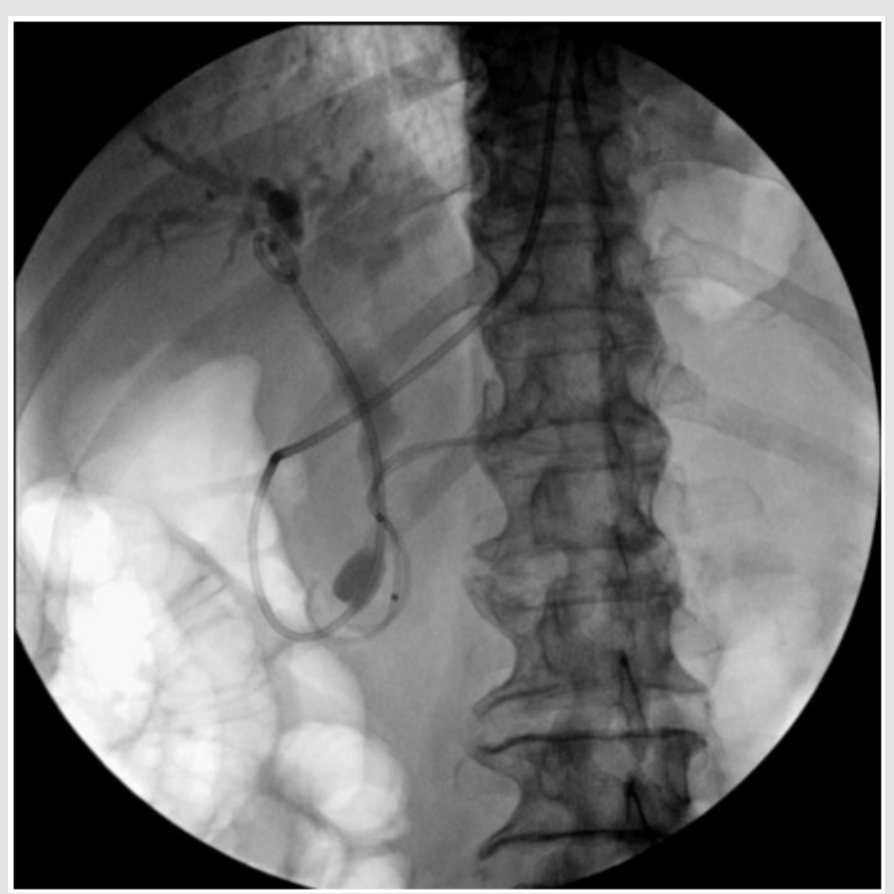

Figure 1: A pancreatic stent migrated proximally into the main pancreatic duct during the first ERCP.

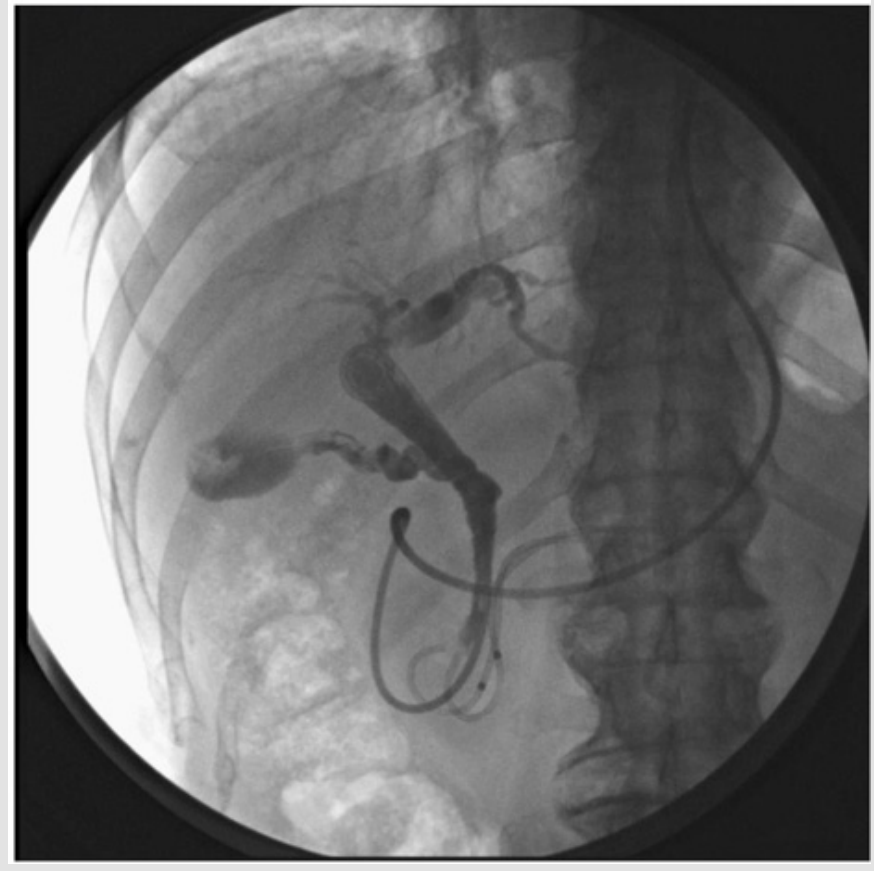

Figure 2: The migrated single pig tail stent is embedded in the pancreatic parenchyma in the head.

\section{Discussion}

PD stent proximal migration is an infrequent complication and can lead to PD injury, recurrent pancreatitis, and even PD leak. Maximal caution is needed during stent placement [1]. Proximal stent migration risk can be minimized by marking the insertion limit or identifying the marker carefully, and avoiding pushing the stent excessively into the main PD. The endoscopist should keep the scope tip a little further away from the papilla to ensure proper stent position during deployment. Once the stent is migrated, the levels of stent migration can be divided into two categories: level 1): retro-papillary migration of the stent, the distal stent end seen within the genu. level 2): the distal end seen beyond the genu [2]. Endoscopic removal can be tailored based on this categorization. Endoscopic retrieval of migrated PD stent has been reported using a variety of devices, such as snares, baskets, tripod grasping forceps, balloons, and forceps. When being seen within the major 
or minor papilla, Level I-migrated stents could be pulled out with a snare forceps or grasping forceps [3]. However, level II-migrated stents may require more advanced techniques such as direct pancreatoscopy for stent retrieval [4]. Endoscopic ultrasoundguided pancreaticogastrostomy for stent removal may fit for a dilated PD [5]. In general, stent removal through ERCP may not be feasible in approximately $3 \%-10 \%$ of patients because of surgically altered anatomy, complete ductal obstructions, or disrupted ducts. In such cases, percutaneous radiologic intervention or surgical treatment may be required, but are associated with relative high morbidity and mortality rates [6].

In this case, because of strenuous peristalsis of the intestine, the proximal stent tip migrated into the main PD first and then penetrated into parenchyma during next 2 months, which resulted in duct distortion and obstruction, and eventually symptomatic pancreatic duct hypertension. The stent migration is different from those two conventional subtypes. This probably explains the initial failure to remove the stent using various devices, including balloon, snare, and basket. These devices could not be advanced into PD due to ductal obstruction. The authors advanced the guide wire to the middle part of the stent and straightened the main PD. Targeting and capturing the migrated and embedded stent must be performed gently using forceps to avoid pushing the stent further into the main PD. After proper capturing of the stent, the endoscopist can remove [7].

\section{Conclusion}

In conclusion, placement of straight and single pig tail pancreatic stent needs maximal caution. In case of proximal stent migration,

ISSN : 2574-1241

DOI: 10.26717/BJSTR.2020.28.004649

Wang Zuozheng MD. Biomed J Sci \& Tech Res

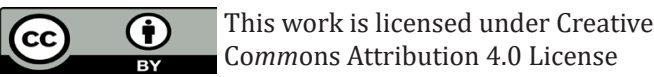

Submission Link: https://biomedres.us/submit-manuscript.php forceps removal can be attempted, especially when the migrated stent is embedded, and the PD is obstructed by the embedded stent.

\section{Acknowledgement}

We gratefully acknowledge Dr. Shou-Jiang Tang for reading the manuscript.

\section{References}

1. Kawaguchi Y, Lin JC, Kawashima Y, Atsuko Maruno, Hiroyuki Ito, et al. (2015) Risk factors for migration, fracture, and dislocation of pancreatic stents. Gastroenterol Res Pract 2015: 36545.

2. Bhandari S, Sharma A, Bathini R, Amit Maydeo (2016) Endoscopic management of internally migrated pancreatic duct stents (with video). Indian J Gastroenterol 35: 91-100.

3. Lu Y, Jin Z, Wu JC, Li-ke Bie, Biao Gong (2015) Endoscopic retrieval technique of proximally migrated pancreatic stents: A retrospective study in a tertiary center. Gastroenterol Res Pract 2015: 485980.

4. Rahimi A, Ejtehadi F (2016) Spy glass pancreatoscopy and successful retrieval of a proximally migrated Pancreatic Stent, Unusual Case and Technical Tips. Middle East J Dig Dis 8: 232-234.

5. Lu L, Jin HB, Yang JF, Zhang XF (2017) Endoscopic ultrasound-guided pancreaticogastrostomy for symptomatic pancreatic duct obstruction caused by migrated pancreatic stent. World J Gastrointest Endosc 9: 535-539.

6. Gonzalez F, Mesleh MG, Lukens FJ, Michael B Wallace, Horacio J Asbun, et al. (2013) Laparoscopic central pancreatectomy and pancreaticogastrostomy for the management of a proximally migrated pancreatic stent. JOP 14: 273-276.

7. Matsubayashi H, Ooka S, Kimura H, Hirokazu Kimura, Toshitatsu Takao, Yuichiro Yamaguchi, et al. (2011) Removal of proximally migrated pancreatic stent using needle knife and capture forceps (with video). J Interv Gastroenterol 1: 90-92.

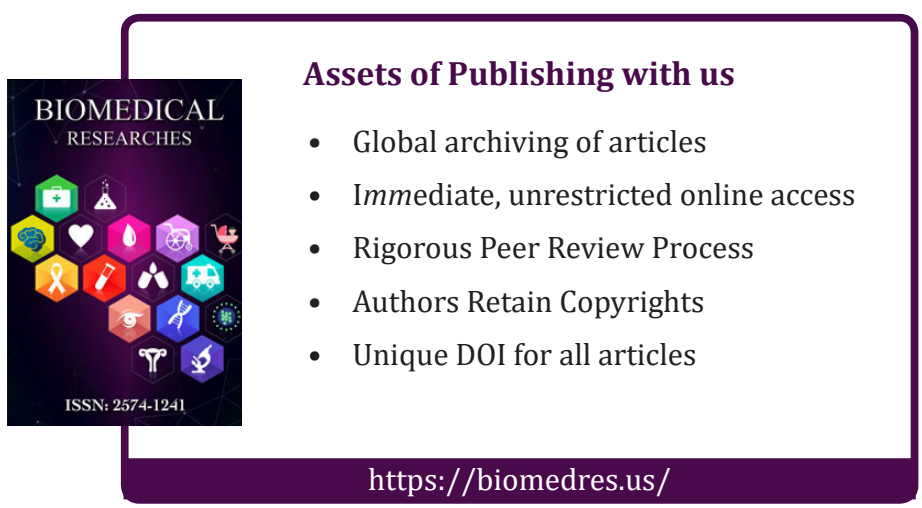

